\title{
¿Cómo explicar matemáticas sin hacer uso de las matemáticas en el contexto de las aulas de mayores? How to explain mathematical contents to senior people but not using maths?
}

\author{
Ana M. Martín Caraballo ${ }^{1}$, Ángel F. Tenorio Villalón ${ }^{2}$ \\ ammarcar@upo.es, aftenorio@upo.es \\ ${ }^{1}$ Departamento de Economía, Métodos \\ Cuantitativos e Historia Económica \\ Universidad Pablo de Olavide \\ Sevilla, España \\ ${ }^{2}$ Departamento de Economía, Métodos \\ Cuantitativos e Historia Económica \\ Universidad Pablo de Olavide \\ Sevilla, España
}

Resumen- La práctica totalidad de las universidades españolas han desarrollado planes de estudios no reglados dirigidos a personas que han superado los 55 años y que bien no pudieron acceder a estudios universitarios bien quieren volver a reengancharse a la vida universitaria. En general, los contenidos a tratar en estas aulas deben estar relacionados con el conocimiento generado y trabajado en el ámbito universitario, pero utilizando un lenguaje y aproximación que evite tecnicismos y academicismos. Esto conlleva un cambio de enfoque a la hora de enseñar matemáticas a este colectivo de estudiantes. En el presente trabajo presentamos algunas de las experiencias que hemos realizado bajo este paradigma, indicando algunas de las problemáticas a las que nos hemos enfrentado para elaborar las sesiones y materiales de trabajo.

\section{Palabras clave: Aula de Mayores; Matemáticas; Universidad}

Abstract- Almost all the Spanish universities have implemented nonregulated study programs for senior people (i.e. 55-year or older people). The goal of these programs is to allow these people to get the opportunity of access to university studies for those that couldn't study in the university or those that want to return again to the university life. In general, contents to be considered for this teaching should be related to the knowledge that university produces, but using a language and approach avoiding technicality and academicism. This leads to change the way to explain and approach mathematics when preparing lectures for this student collective. In this paper, we present some experiences that were performed under this teaching paradigm when explaining mathematics to senior people, commenting some of the difficulties to be faced during the preparation of the lectures and materials.

\section{Keywords: Senior-people classroom; Mathematics; University}

\section{INTRODUCCIÓN}

En general, las universidades españolas han integrado entre su oferta de estudios programas no reglados que se ofertan a personas que han superado los 55 años. Este es el único requisito pedido ya que no se exige que tengan por ejemplo, un nivel de estudios determinado, pero sí que quieran vivir la experiencia de la vida universitaria.

Es a partir de los años 60 del siglo pasado cuando comienza a plantearse la necesidad de llevar a cabo políticas sociales a favor de las personas mayores, que por supuesto, se desarrollan a distinto ritmo en función de las peculiaridades de cada país. Tradicionalmente, la atención a las personas mayores se ha estructurado en torno a las pensiones, la asistencia sanitaria y los servicios sociales; pero a partir de 1982 y después de la I Asamblea Mundial sobre el Envejecimiento, una de sus recomendaciones es la de establecer programas de educación mediante la asignación apropiada de recursos y con contenidos de enseñanzas que sean útiles y estén adaptadas a las necesidades de las personas mayores, de esta forma, se enlaza también con el concepto de la UNESCO sobre educación permanente.

Frente a un constante aumento de la esperanza de vida de la población, al aislamiento socio-cultural en el que el colectivo de personas mayores está y por supuesto, con el compromiso social de llevar a cabo políticas de bienestar social y ya que el aprendizaje se entiende como un proceso permanente que se lleva a cabo a lo largo de toda la vida, la universidad no debe ser ajena a todo esto. Por ello, a través de la educación y de la formación, la universidad debe romper el aislamiento social, y favorecer la autonomía de los mayores y por supuesto, las relaciones intergeneracionales.

Así, por estos motivos mencionados anteriormente, hace ya años comenzaron movimientos de creación de las Universidades de la Tercera Edad. Esta tendencia es recogida tanto por el Plan Gerontológico Nacional como en el Plan Andaluz de Servicios Sociales y, concretamente, en el art.31 de la Ley 6/1999 de 7 de julio, de atención y protección de las personas mayores, donde se establece que, para la formación de las personas mayores, se promoverá la organización por parte de las Universidades andaluzas de aulas de formación, cursos de perfeccionamiento y otras actividades formativas dirigidas a personas mayores, con independencia del nivel académico obligatorio para acceder a la educación en determinadas materias, y sin que ello comporte la obtención de una titulación académica reglada.

Una vez dentro de estas aulas de mayores, lo primero a destacar es que los contenidos a tratar deben estar relacionados con el conocimiento generado y trabajado en el ámbito universitario, pero dado el carácter heterogéneo del aula en el que se imparte la enseñanza, es necesario utilizar un lenguaje que evite tecnicismos y academicismos. Por todo esto, cuando 
se intenta enseñar matemáticas conlleva obligatoriamente un cambio de enfoque a la hora de enseñar a este colectivo.

Desde hace ya más de ocho años los autores de este trabajo participamos en el Aula de Mayores de nuestra universidad y nuestro objetivo es presentar en este trabajo algunas de las experiencias que hemos realizado bajo este paradigma, indicando además, algunas de las problemáticas a las que nos hemos enfrentado para elaborar las sesiones y materiales de trabajo.

\section{CONTEXTO: LAS AULAS DE MAYORES}

Como se indicó anteriormente, en las décadas de los 50 y 60 del siglo pasado surgieron los primeros movimientos de creación de las Universidades de mayores y de la Tercera Edad en Europa y, más concretamente, en Francia (Velázquez y Fernández, 1998). Esta tendencia se recoge en la actualidad tanto en el Plan Gerontológico Nacional (IMSERSO, 1993) y el Plan de Acción para las personas mayores 2003-2007 (IMSERSO, 2003) como en el Plan Andaluz de Servicios Sociales (Junta de Andalucía, 1989). Es más, a nivel de la Comunidad Autónoma de Andalucía, la organización por las universidades andaluzas de actividades formativas para las personas mayores será promovida por las Administraciones Públicas según recoge la Ley 6/1999 en su artículo 31. Dicha formación podrá ser mediante aulas de formación, cursos de perfeccionamiento $\mathrm{u}$ otras actividades dirigidas a estas personas, con independencia del nivel académico obligatorio para el acceso a la educación universitaria y sin que comporte la obtención de una titulación académica reglada.

A nivel mundial, las oportunidades educativas que las universidades deberían ofertar a las personas mayores se recogen en UNESCO (1998), donde se enfatiza la necesidad prioritaria de una apertura de los centros universitarios a los adultos mediante "oportunidades" de aprendizaje "flexibles, abiertas y creativas” (Fedriani y Tenorio, 2006).

En la ponencia-marco del VI Encuentro Nacional de Programas Universitarios para Mayores (véase Bru, 2002), se establece que una de funciones de las Universidad Española relativa a extensión cultural es la organización de aulas de mayores. Además, deben tenerse en cuenta dos detalles que subrayan también el interés que tiene la Universidad en los mayores. El primero es el envejecimiento de España, que a mediados del siglo XXI será probablemente el país más envejecido del planeta, con lo que los mayores de 50 serán quienes mantengan las estructuras económicas y de financiación de la Universidad. En segundo lugar, la jubilación va retrasándose debido a las políticas europeas actuales, por lo que los profesionales han de reciclar sus conocimientos de forma casi obligada.

En Fedriani y Tenorio (2006) se indica que en cuanto a la temática tratada en los programas universitarios dirigidos a mayores, se observa un mayor porcentaje de asignaturas de Humanidades y Ciencias Sociales, aunque en dichos programas suelen existir cinco áreas de conocimiento bien diferenciadas que son: a) Humanidades y Ciencias Sociales, b) Ciencias Jurídicas y Económicas, c) Ciencias de la Tierra y el Medio Ambiente, d) Ciencias Biosanitarias y por último, e) Ciencias Tecnológicas. De hecho, en los planes de estudio de las diversas Aulas de Mayores o de la Experiencia que existen en España se observa un porcentaje muy superior de los bloques a) y b), respectivamente, siendo el menor porcentaje el del bloque e). Nótese que todas estas actividades caen en el ámbito de la Enseñanza no reglada y suelen depender del Vicerrectorado que tienen entre sus funciones las actividades de Promoción Cultural y Extensión Universitaria. Sin embargo, existen universidades específicamente destinadas a la docencia de adultos, una de las que más tradición tiene en nuestro país, es la Universidad de la Tercera Edad (UTE) y en ella, sus titulaciones son todas referentes a los ámbitos de conocimientos relativos a los bloques a) y b).

Las Aulas de Mayores se organizan dentro de las propias universidades de dos formas bien diferenciadas. La primera sigue el modelo tradicional, es decir, los alumnos van a la universidad con el objetivo de recibir sus clases, mientras que en el segundo modelo es la universidad la que "se desplaza" a la localidad donde estén los alumnos.

\section{A. El Aula de Mayores en la Universidad Pablo de Olavide}

En este apartado se describe el modelo del Aula de Mayores en la Universidad Pablo de Olavide de Sevilla.

Para el curso académico 2002/03, la Universidad Pablo de Olavide de Sevilla (en adelante, UPO) puso en marcha un programa de formación científica, cultural y social que perseguía mejorar la calidad de vida de las personas mayores y fomentar su participación como dinamizadores sociales. Este programa, denominado Aula Abierta de Mayores, está dirigido a mayores de 55 años y sigue las directrices comentadas en la sección anterior referentes a la Educación de Mayores (Memoria Evaluativa Curso 2003-2004).

A diferencia de otras universidades, el Aula Abierta de la Universidad Pablo de Olavide se puso en práctica en municipios y comarcas de Sevilla cuyas distancias a la capital dificulta a las personas mayores el acceso a la formación y participación en ámbitos universitarios. Actualmente, son diecisiete las sedes del Aula Abierta, las citadas sedes son: Alcalá de Guadaíra, Aznalcóllar, Bormujos, Castilleja de la Cuesta, Gerena, Gilena, Gines Herrera, La Puebla de Cazalla, La Puebla del Río, Lebrija, Mairena del Alcor, Pedrera, Pilas, Salteras, Santiponce y Tomares .

El único requisito que se les pide a los participantes en el Aula Abierta es que posean interés por aprender y conocer cosas nuevas. Es por ello que no se requiere titulación para asistir, con lo que los contenidos son tratados a un nivel divulgativo que pueda ser entendido por cualquier persona y por tanto, en las sesiones del Aula Abierta, no se utiliza un lenguaje tan formal como en las clases de la formación reglada impartidas tradicionalmente en la universidad.

En la UPO, el Aula Abierta de Mayores tiene un programa formativo estructurado en tres cursos académicos (en cada uno de los diecisiete municipios donde se imparte), cada uno de los tres cursos cuenta con unas 100 horas lectivas. En cada curso se trabajan tres bloques de conocimientos distintos, que son: Ciencias Sociales, Científico-Técnico y Jurídico-Económico. Los cursos que se realizan, comprenden tanto sesiones lectivas como conferencias. Destacar que aunque como se ha indicado anteriormente el Aula Abierta de la UPO se imparte en los distintos municipios, los alumnos se desplazan al campus de la UPO en al menos tres ocasiones durante el curso para recibir allí ciertas sesiones y de esta forma, hacer que se sientan parte e integrados en la universidad. 
Concluimos la presente sección indicando cómo se financia esta actividad del Aula de Mayores y cuáles son las instituciones que participan, además de la UPO, en la realización de esta actividad. De hecho, el origen de este aula está en la colaboración entre varias instituciones y organismos sociales, los cuales actúan sobre el ámbito educativo y la protección de las personas mayores. En resumen, el Aula Abierta de Mayores de la UPO se financia con las aportaciones de los distintos Ayuntamientos que demandan dicha actividad, la Diputación Provincial de Sevilla, la Consejería de Asuntos Sociales y las aportaciones de las cuotas de los alumnos participantes. Por su parte, la UPO contribuye aportando los recursos organizativos y de gestión, además, por supuesto, del apoyo administrativo general. En cuanto a los gastos de docencia y de funcionamiento específico de cada programa, éstos serán subvencionados mediante convenio con las instituciones anteriormente citadas (Fedriani y Tenorio, 2006).

\section{DESCRIPCIÓN: EXPERIENCIAS DOCENTES EN MATEMÁTICA}

Los autores de este trabajo, llevan ocho años participando en el Aula de Mayores de la Universidad Pablo de Olavide. En estos años, hay que destacar que la estructura de las clases ha cambiado. Al principio, casi todo lo que se demandaba eran conferencias sobre temas específicos, mientras que desde hace ya tres cursos, las sesiones se estructuran en módulos que se imparten en varias sesiones sobre un tema común.

Hay que destacar que el principal objetivo que tienen los autores de este trabajo para participar en el Aula de Mayores es el de divulgar las matemáticas y hacer que un público muy particular, y a la vez heterogéneo, se interese en éstas, por tanto, la máxima que se persigue es motivar a los alumnos, hacerles parte activa del conocimiento y usar sus experiencias vitales en las diversas sesiones para que se interesen en las matemáticas y sobre todo, le pierdan el miedo a éstas. Por otra parte, la heterogeneidad en la formación de los asistentes es significativa. Por todo esto, se utiliza como metodología para las sesiones una que no sea puramente expositiva y en la que los asistentes puedan participar en el desarrollo de la misma de manera continuada y activa, buscando su motivación constantemente.

En general, las matemáticas suelen ser una de las asignaturas que más problemas plantea a los estudiantes a lo largo de su vida académica, por lo que nuestro principal objetivo es cambiar esta apreciación durante las sesiones del Aula de Mayores. Por tanto, lo que nos proponemos mostrar a estos grupos de alumnos tan heterogéneos, es que las matemáticas pueden ser divertidas. Con tal fin, realizamos sesiones de "Magia y Matemática" o de "Humor y Matemática”, por otra parte, también queremos que los alumnos vean que las matemáticas pueden "hacerse” y para ello, impartimos varios módulos con contenidos de matemáticas manipulativas como "Matemáticas con Arte y Arte con Matemáticas", "Puzzles", "El problema del vigilante”, etc. Por otra parte, para lograr que los alumnos también vean las matemáticas como algo útil para ellos, se imparten módulos de "Matemáticas para la vida cotidiana", "Sistemas de numeración", "Una banda muy especial", "Papiroflexia y fractales”, "Astronomía”, etc. Por ultimo, en algunos módulos se intenta relacionar las matemáticas con otras disciplinas como la historia o la religión así ocurre por ejemplo, en el módulo de "El cero, el infinito y la nada”.

Volviendo al rechazo y al pánico e incluso horror que tienen los estudiantes a las matemáticas, hay al menos tres razones importantes por las que esto puede suceder en el ámbito del Aula de Mayores:

I. El tipo de tarea que se propone a los estudiantes: se incide más en la comprensión de los conceptos y no tanto en la exactitud y rapidez de computación.

II. La desvinculación de las matemáticas con los problemas de la vida real: esto radica en que los símbolos y reglas formales se enseñan como si se trataran de convenciones arbitrarias y no como expresiones de regularidades y relaciones fundamentales entre cantidades y entidades físicas.

III. La separación existente entre aprendizaje y enseñanza, es decir, la falta de información por parte del profesor de los conocimientos que poseen los alumnos $\mathrm{y}$, sobre todo, de la naturaleza propia del conocimiento del alumnado.

Con nuestra experiencia como docentes en el Aula de Mayores podemos afirmar que los alumnos de este aula son por lo general muy exigentes, receptivos y activos. Suelen implicarse mucho más que los alumnos universitarios por lo que participar en el Aula de Mayores, es siempre una experiencia muy gratificante para el docente. Además, los alumnos demandan una docencia que se centre en la práctica cotidiana. Se interesan por todo lo que les enseñan y demandan información y materiales del tema que se está tratando. Otras veces, de una sesión para la siguiente, los alumnos traen materiales o información que han encontrado relacionado con los temas tratados en sesiones anteriores y lo comentan con los profesores.

En Vila (2002) se indican diversas características referentes al alumnado que participa en estos programas. Por una parte, suelen ser personas que están mayoritariamente inactivas. Por otro, los niveles educativos de los participantes son muy heterogéneos, predominando los que tienen estudios sólo hasta Educación Secundaria y a veces sólo hasta Primaria. Por tanto, es importante y además necesaria, la atención a la diversidad en el aula.

En el VI Encuentro Nacional de Programas Universitarios para Mayores Bru (2002) se obtuvieron varias conclusiones que creemos resultan de interés a la hora de afrontar la enseñanza con personas mayores:

- Una persona puede aprender a cualquier edad; solo depende de su voluntad.

- La formación personal ha de primar ante fines competitivos o profesionales. Éste es uno de los principales objetivos de las Aulas de Mayores.

- $\quad$ La capacidad de adaptación debe fomentarse a cualquier edad. Es decir, los mayores deben permanecer activos y deben poder adaptarse a las nuevas situaciones que se presentan.

- Los cursos son gratificantes para los alumnos, ayudando a la realización personal y al fomento de las relaciones interpersonales $y$ la 
convivencia, tanto entre los propios alumnos como entre alumnos y profesores universitarios.

Los cursos facilitan la comprensión de la realidad actual, creando estímulos a los alumnos, para mantenerlos activos física y psíquicamente y para alcanzar las competencias necesarias sobre salud, ocio y vida cotidiana.

\section{RESUltados. DifiCUltades EN LA ELABORACiÓN DE MATERIALES}

Existen diferentes materiales y recursos para el aprendizaje de las matemáticas, desde los más clásicos como el profesor, libro, pizarra, cuaderno, etc.; los más populares, aunque no por ello los más utilizados como calculadora, reglas, regletas, puzzles, pentominos, etc.; hasta otros más accesibles y cercanos como palillos, menús de restaurantes, folletos publicitarios de tiendas, etc.. Los materiales en la clase de matemáticas son un medio, no son un fin en sí mismos, es decir, hay que conseguir el aprendizaje de las matemáticas a través de los materiales pero éstos no deben ser el objetivo de aprendizaje.

Utilizar materiales didácticos y juegos adecuados permiten entre otras cosas, mejorar la actitud de los alumnos ante las matemáticas, desarrollar la creatividad, mostrar que existen problemas que no tienen una solución determinada de antemano, hacer matemáticas adaptadas a las posibilidades de cada alumno del grupo; esto último es importante en el contexto del Aula de Mayores ante la heterogeneidad que nos encontramos en los grupos de alumnos.

En las sesiones del Aula de Mayores es interesante proponer una serie de materiales didácticos, con diferentes actividades, para que nos sirvan de referencia y nos permitan utilizarlos como apoyo en nuestra clase. Por ejemplo, en la sesión de "Juegos y matemáticas" se reparten a los alumnos diferentes juegos como laberintos, sudokus, etc., o en una parte de la sesión de "Matemáticas útiles para la vida cotidiana”, se introducen los gráficos y cómo analizarlos, para ello se les proporciona a los alumnos el gráfico de la Figura 1 y en primer lugar se les introduce el uso de los gráficos como medio para presentar información desde un punto de vista histórico para pasar a intentar interpretar entre todos el gráfico de la Figura 1 que hay quien lo considera el mejor mapa estadístico de la historia porque con bastante habilidad el autor (que era ingeniero) fue capaz de agrupar en una sola imagen todos los datos como son: a) Geografía: ríos, ciudades y batallas, b) Camino seguido por el ejército, c) Dirección del ejército hacia Rusia (marrón) y la vuelta (en negro), d) Número de soldados restantes (lo da el grosor de las líneas), e) Temperatura y f) Tiempo (cronología).

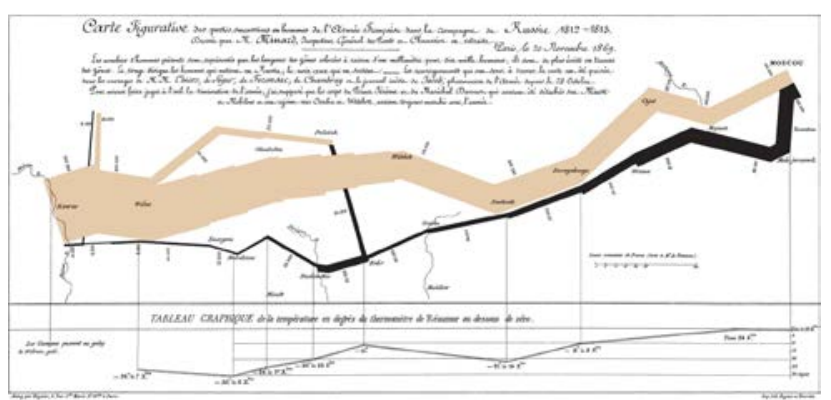

Figura 1. Gáfico de Minard.
En otras sesiones, por ejemplo, para introducir los sistemas de numeración, se les presenta éstos en diferentes civilizaciones para por último, dar el concepto de sistema de numeración, los tipos que hay y estudiar más a fondo el utilizado hoy día (ver Figura 2).

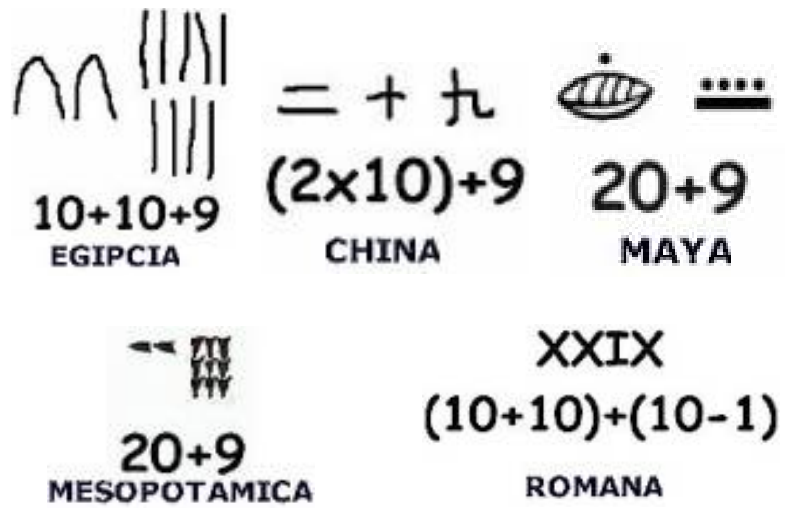

Figura 2. El número 29 en diferentes sistemas de numeración.

Algunos de los puzles utilizados en la sesión dedicada a ellos pueden verse en la Figura 3.

Figura 3. Puzzles.
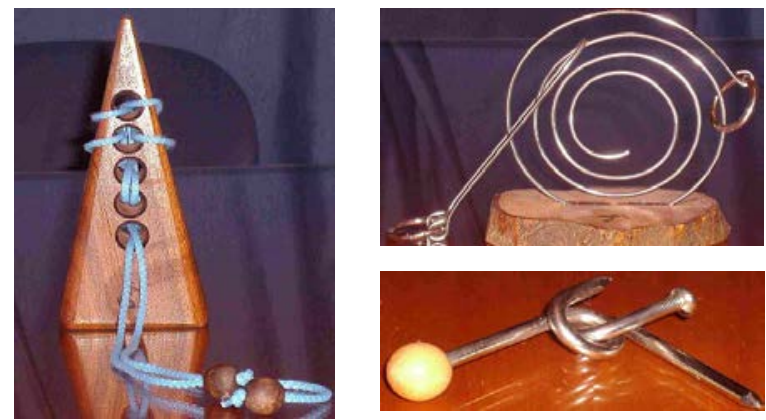

Las mayores dificultades que nos encontramos para la elaboración de materiales, sobre todos los expositivos, son la necesidad de no utilizar el lenguaje formal de las matemáticas ya que si no lo hiciéramos así, la mayoría de los alumnos no podrían seguir nuestras clases, por lo que hay que utilizar un lenguaje más cercano para ellos, es decir, utilizamos el lenguaje natural y no el lenguaje matemático. A lo largo de estos cursos, hemos podido elaborar gran cantidad de materiales para los alumnos del Aula de mayores que tienen a su disposición en la página web del Aula de Mayores de la Universidad Pablo de Olavide.

\section{CONCLUSIONES}

Lo primero que es necesario destacar, es que participar en el Aula Abierta de Mayores es una experiencia muy gratificante para el docente. Ésto es así principalmente porque los alumnos van predispuestos a aprender y se muestran activos en clase, aportando sus experiencias, sus ideas y sus dudas.

Por otra parte, es importante que el docente elija temas para las sesiones que no precisen de muchos requisitos previos de matemáticas. Es interesante y gratificante, elegir temas que estén relacionados con el entorno de los alumnos o con la vida cotidiana de nuestros alumnos y que por tanto, les resulten de bastante interés y utilidad. De esta forma, pueden ver la relación entre matemáticas y vida real, además, de poder darse 
cuenta de lo necesaria que son las matemáticas en el día a día de los alumnos, de cómo, sin darse cuenta, la utilizan diariamente.

Con este tratamiento hay muchos temas de contenidos matemáticos que se pueden tratar, incluso, es posible encontrar temas de problemas cercanos a estos alumnos y que aún en la actualidad, son problemas abiertos $y$ no resueltos en matemáticas.

\section{REFERENCIAS}

Bru, C. (ed.) (2002). Los Modelos Marco de Programas Universitarios para Mayores. Actas del VI Encuentro Nacional de Programas Universitarios para Mayores. Universidad de Alicante.

Fedriani, E.M. y Tenorio, A.F. (2006). Geometría para alumnos mayores de 55 años: una experiencia en un Aula de Mayores. Revista de Educación Matemática EPSILON, 65 (2).

IMSERSO (1993). Plan Gerontológico Nacional. Madrid.

IMSERSO (2003). Plan de Acción para las personas mayores 2003-2007. Consejo de Ministros de 29 de agosto. Disponible en http://segsocial.es/imserso/normativas/planppmm20032007.pdf.

Junta de Andalucía (1989). Plan Andaluz de Servicios Sociales. Boletín Oficial de la Junta de Andalucía (BOJA) de 12 de abril.
Ley 6/1999, de 7 de julio, de Atención y Protección a las Personas Mayores. Boletín Oficial de la Junta de Andalucía $n^{\circ}$ 87, de 29 de julio. Disponible en http://juridicas.com/base_datos/CCAA/an-16-1999.html

UNESCO (1998). Declaración Mundial sobre la Educación Superior en el s.XXI. Disponible en http://www.unesco.org/education/educprog/wche/declara tion_spa.htm

Universidad Pablo de Olavide. Página Web del Aula Abierta de Mayores; http://www.upo.es/general/centros_depart/otros_centros/ aula_mayores/otros_amayores.html

Vicerrectorado de Promoción Social y Extensión Universitaria de la Universidad Pablo de Olavide (2004). Aula Abierta de Mayores. Memoria Evaluativa Curso 2003-2004. Disponible en http://www.upo.es/general/centros_depart/otros_centros/ aula_mayores/docu/memoria2004_aam.pdf.

Velázquez, M. y Fernández, C. (1998). Las Universidades de mayores. Una aventura hecha realidad. Secretariado de Publicaciones de la Universidad de Sevilla.

Vila, D. (2002). Las Aulas de la Experiencia. Muf@ce. Revista electrónica $n^{\circ}$ 18/188. Disponible en http://www.map.es/gobierno/muface/o188/educ.htm 